\title{
Non-Gaussian curvature distribution of actin-propelled biomimetic colloid trajectories
}

\author{
Stephan Schmidt • Jasper van der Gucht • \\ P. Maarten Biesheuvel - Richard Weinkamer · \\ Emmanuèle Helfer · Andreas Fery
}

Received: 27 February 2008/Revised: 18 April 2008/Accepted: 18 April 2008/Published online: 20 May 2008

(C) The Author(s) 2008

\begin{abstract}
We analyze the motion of colloids propelled by a comet-like tail of polymerizing actin filaments. The curvature of the particle trajectories deviates strongly from a Gaussian distribution, implying that the underlying microscopic processes are fluctuating in a non-independent manner. Trajectories for beads of different size all showed the same non-Gaussian behavior, while the mean curvature decreased weakly with size. A stochastic simulation that includes nucleation, force-dependent dissociation, growth, and capping of filaments, shows that the non-Gaussian curvature distribution can be explained by a positive feedback mechanism in which attached chains under higher tension are more likely to snap.
\end{abstract}

Keywords Actin · Propulsion - Trajectory analysis · Stochastic simulation

S. Schmidt and J. van der Gucht contributed equally to this paper.

S. Schmidt · A. Fery $(\bowtie)$

Department of Physical Chemistry II, University of Bayreuth, Bayreuth, Germany

e-mail: andreas.fery@uni-bayreuth.de

J. van der Gucht

Laboratory of Physical Chemistry and Colloid Science,

Wageningen University, Wageningen, The Netherlands

e-mail: jasper.vandergucht@wur.nl

P. M. Biesheuvel · R. Weinkamer

Max Planck Institute of Colloids and Interfaces,

Potsdam, Germany

E. Helfer

Laboratoire d'Enzymologie et Biochimie Structurales,

CNRS, Gif-sur-Yvette, France

\section{Introduction}

The ability to generate forces and move actively is a key feature of many micro-organisms and eukaryotic cells and nature has found various pathways to accomplish it. For example, the intracellular movement of bacterial pathogens, such as Listeria monocytogenes, is driven by polymerization of actin:actin filaments are nucleated at the bacterial surface and their growth by monomer insertion generates a force that pushes the bacterium forward (Theriot et al. 1992). Most of the proteins responsible for Listeria movement have also been found in the lamellipodium of crawling cells, making Listeria a popular model system for actin-based cell motility (Pantaloni et al. 2001). The actin-propelled motion of Listeria is also displayed by some organelles and liposomes and it can be reproduced using simple model systems, such as phospholipid vesicles (Upadhyaya et al. 2003; Giardini et al. 2003), oil droplets (Boukellal et al. 2004), and polystyrene beads (Cameron et al. 1999, 2004; Loisel et al. 1999; Wiesner et al. 2003; Paluch et al. 2006). These biomimetic systems have been very useful for investigating the biochemical and biophysical details of actin-based movement. Nevertheless, it still remains poorly understood how the polymerization kinetics of many individual actin filaments are coordinated to generate directed motion.

A relatively overlooked aspect of actin-based movement, with very important consequences for bacterium spreading and efficient cargo delivery, is the directionality of the movement. How does the bacterium or particle change its direction and what trajectory does it follow? Understanding the shape of the trajectory also gives important insight in the underlying microscopic processes and their regulation (Cameron et al. 2004). Listeria typically follows very regular, periodic trajectories, that have 
been explained recently with a phenomenological kinematic model (Shenoy et al. 2007). Actin-propelled beads follow more random trajectories, that have not been analyzed in great detail. A theoretical study considers the curvature of the comet tail resulting from random variations in the locations of pushing actin filaments (Rutenberg and Grant 2001). The local curvature is predicted to have a Gaussian probability distribution, but this distribution has not been measured experimentally. In this paper, we present a statistical analysis of the trajectories of a large number of actin-propelled beads. We show that the curvature distribution deviates significantly from a Gaussian distribution, which indicates that the microscopic processes that determine the trajectories are not governed by simple random statistics. A stochastic model is proposed to explain these observations.

\section{Materials and methods}

We use polystyrene beads with radii of $1,1.5$, and $3 \mu \mathrm{m}$, coated with N-WASP, an activator of the actin-nucleating Arp2/3 complex (for coating procedure, see Wiesner et al. 2003). The beads are placed in a motility medium containing the proteins necessary for actin-based movement (Loisel et al. 1999): $7 \mu \mathrm{M}$ actin, $9 \mu \mathrm{M} \mathrm{ADF} /$ cofilin, $0.1 \mu \mathrm{M}$ gelsolin, $2.4 \mu \mathrm{M}$ profilin, $0.1 \mu \mathrm{M}$ Arp $2 / 3$ complex. The medium is supplemented with $10 \mathrm{mg} / \mathrm{ml}$ BSA to prevent non-specific adsorption, $0.2 \%(\mathrm{wt} / \mathrm{vol})$ methylcellulose to minimize Brownian motion of the colloids, and $2 \mathrm{mM}$ ATP as the fuel. Samples ( $8 \mu \mathrm{l}$ for $3 \mu \mathrm{m}$ beads, $5 \mu \mathrm{l}$ for 1 and $1.5 \mu \mathrm{m}$ beads) were placed between a glass slide and coverslip $(20 \times 20 \mathrm{~mm})$, such that the sample height was at least twice the bead diameter. Note that the bead velocity may nevertheless be affected slightly by the surfaces (Shaevitz and Fletcher 2007). Beads were observed using an inverted Zeiss axiovert 200 microscope equipped with a $20 \times$ phase contrast objective (N.A. 0.4) and an Axiocam HRm camera, giving images with a pixel size of $170 \mathrm{~nm}$. Images were captured at 30-s intervals for several hours. Individual particles were tracked using an algorithm that finds the center of mass of each particle in successive images, with a spatial accuracy of a few tens of nanometers (Carter et al. 2005).

\section{Results and discussion}

After a stage of homogeneous growth, the actin shell breaks open (van der Gucht et al. 2005) and the beads start moving in curved trajectories, propelled by a comet tail of actin filaments (Fig. 1a-d). The speed of the beads is on the order of $2.5 \mu \mathrm{m} / \mathrm{min}$ for beads with a radius of 1 and $1.5 \mu \mathrm{m}$, while beads of $3 \mu \mathrm{m}$ radius move more slowly at approximately $1.2 \mu \mathrm{m} / \mathrm{min}$. After several hours the speed of the beads starts to decrease gradually, and
Fig. 1 Phase contrast images of $1 \mu \mathrm{m}(\mathbf{a}), 1.5 \mu \mathrm{m}(\mathbf{b})$, and $3 \mu \mathrm{m}$ (c) radius beads. Scale bar: $30 \mu \mathrm{m}$. About $80 \%$ of the tails are hollow for $R=3 \mu \mathrm{m}$, while for $R=1$ and $1.5 \mu \mathrm{m}$, all tails are homogeneous. Note that the actual trajectories are much longer, because the tail depolymerizes from the back. d Experimental and e simulated trajectories for three bead sizes. Time taken for trajectories is $120 \mathrm{~min}$ for $1 \mu \mathrm{m}$ beads and $300 \mathrm{~min}$ for 1.5 and $3 \mu \mathrm{m}$ beads
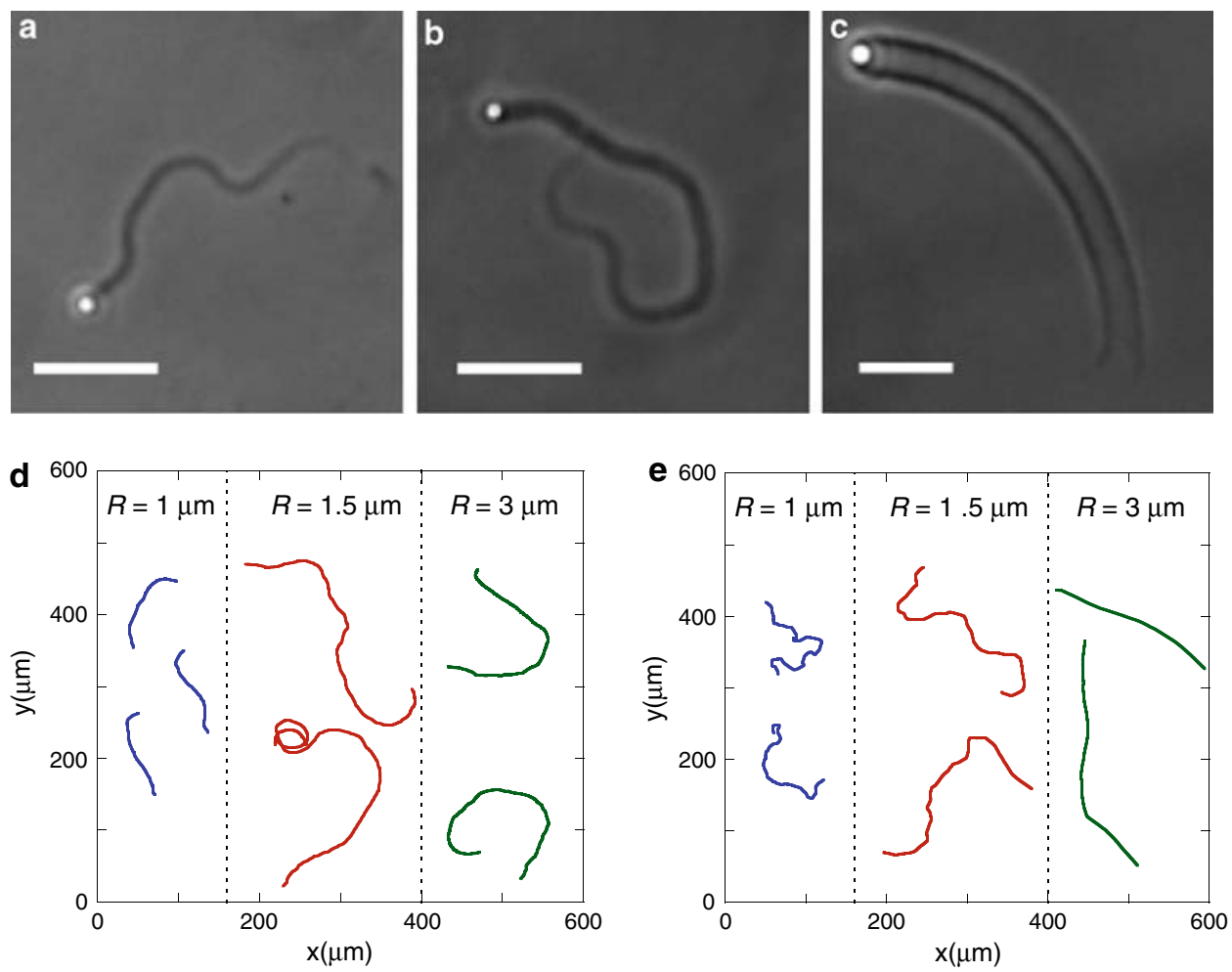
after $8 \mathrm{~h}$ it is about 20 percent of the initial speed. To characterize the particle trajectories, we analyze the local curvature $\kappa$ of the trajectories, which can be calculated as $\kappa=(\ddot{x} \ddot{y}-\ddot{y} \ddot{x})\left(\dot{x}^{2}+\dot{y}^{2}\right)^{-3 / 2}$, where $x$ and $y$ are spatial coordinates and each dot denotes differentiation with respect to time $t$ (Kreyszig 1991). In order to avoid possible artifacts due to errors in the position determination and amplification thereof by the numerical differentiation of the $x y$ data, the trajectories were first numerically smoothed using a least squares polynomial smoothing procedure, in which a quadratic function was fitted locally to the data in a moving window corresponding to a path length of $10 \mu \mathrm{m}$. In this way, only radii of curvature larger than roughly $1 \mu \mathrm{m}$ are considered, much larger than the error in the position determination (a few tens of nanometers, see "Materials and methods"). Figure 2a shows a plot of the measured (smoothed) curvature at each time frame as a function of the corresponding velocity for all three bead sizes. Clearly, there is no correlation between the two. As shown in Fig. 2b (blue squares), the rootmean-square curvature decreases slightly with increasing bead size, from $0.13 \mu \mathrm{m}^{-1}$ for $R=1 \mu \mathrm{m}$ to $0.08 \mu \mathrm{m}^{-1}$ for $R=3 \mu \mathrm{m}$. We then construct the probability distribution $P(\kappa)$, where $P(\kappa) \mathrm{d} \kappa$ gives the relative frequency of curvatures between $\kappa$ and $\kappa+\mathrm{d} \kappa$, and the cumulative distribution function $\operatorname{CDF}(\kappa)$, obtained by ranking the absolute values of the experimental $\kappa$ from low to high and plotting their ranking number (divided by the total number of data points) as a function of $\kappa$. We note that changing the window size or order of the smoothing filter or using a cubic splines algorithm did not change the shape of the curvature distribution, although the absolute values of $\kappa$ shift towards smaller curvatures if a larger smoothing window is used (doubling the window size decreases $\langle\kappa\rangle$ by about $10-20 \%)$. Also, restricting the analysis to the first hours of the trajectories, where the speed is more or less constant, did not affect the shape of the curvature distribution.

Figure $2 \mathrm{c}$ and $\mathrm{d}$ show the measured $P(\kappa)$ and $\operatorname{CDF}(\kappa)$. After normalizing the curvature by the root-mean-square curvature, the data for different radii all collapse onto one curve (apart from small deviations for the smallest beads at small curvatures). The dashed black lines in Fig. 2c and d are fits to a Gaussian distribution, as predicted by Rutenberg and Grant (2001). For small curvatures, the Gaussian distribution fits the experimental data reasonably well, but for larger curvatures there are strong, systematic deviations for all three bead sizes: large curvatures occur much more frequently than would be expected for a Gaussian distribution. (An empirical formula that gives a rather accurate description of the experimental distribution is a modified log-normal distribution, $P(\kappa)=\mathrm{cst} \cdot \exp [-(\ln (a|\kappa| /\langle\kappa\rangle+$ $\left.1))^{2}\right]$, with $a \approx 2.32$.) We aim at developing a microscopic model that naturally reproduces these observations.

According to the central limit theorem, a combined effect of many additive random processes always results in a
Fig. 2 Results of trajectory curvature analysis. a Local curvature as a function of bead velocity for different radii. Lines are moving averages (period of 250 points). b Rootmean-square curvature as a function of bead radius: experimental (exp), simulations (sim1), and simulations with a nucleation probability that decreases exponentially with increasing distance from the tail edge (see text) $(\operatorname{sim} 2)$. c Curvature probability distribution and $\mathbf{d}$ cumulative curvature distribution function for different radii. The curvatures have been normalized with respect to the root-mean-square curvature.

The dashed black lines correspond to a Gaussian distribution and the solid black lines to an empirical, modified log-normal distribution (see text)
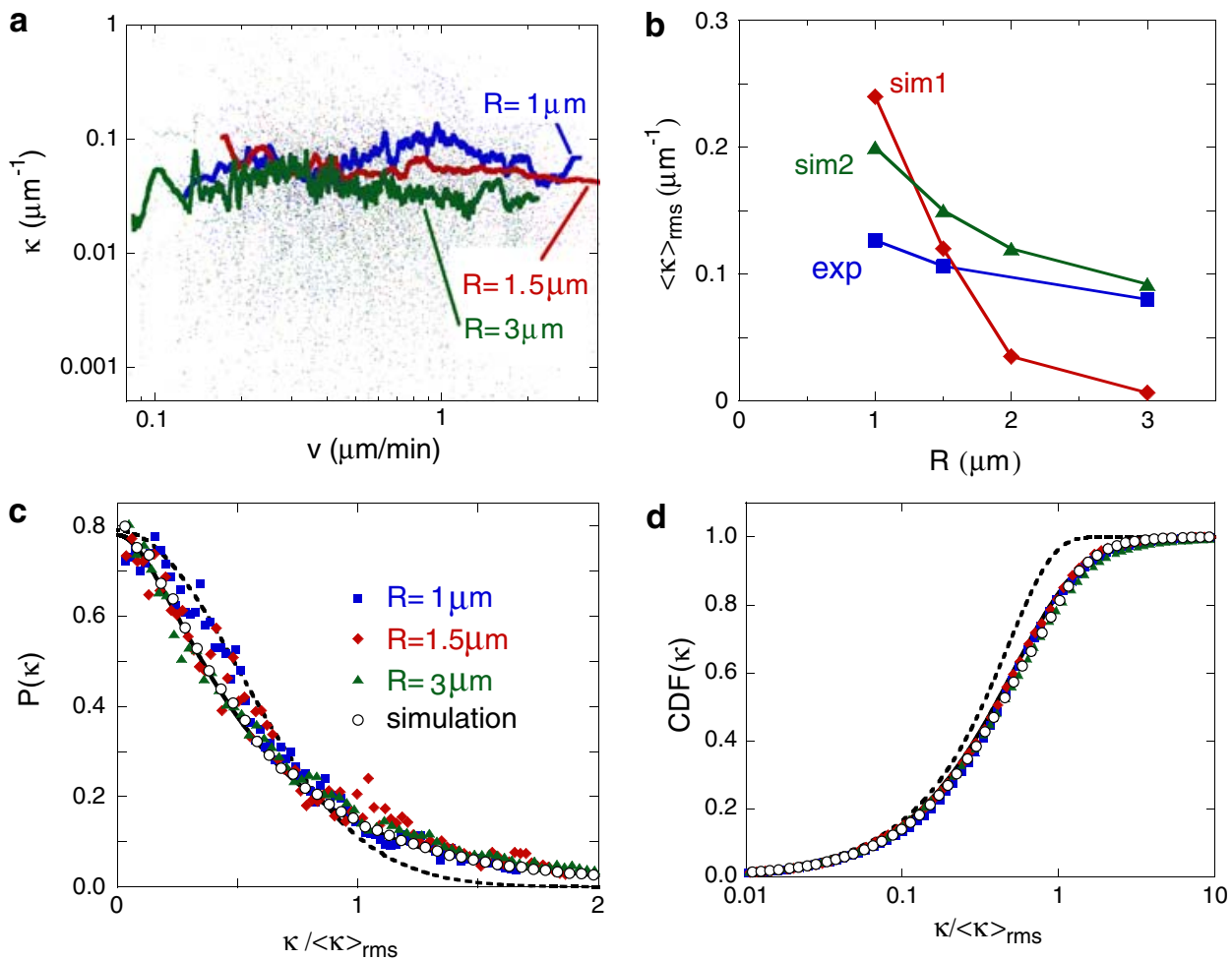
Gaussian distribution. Therefore, the non-Gaussian distribution of curvatures can not be explained by models that derive curved trajectories from independent random variations in microscopic parameters (such as the local filament density, as in Rutenberg and Grant (2001). To explain the relatively high probability of finding large curvatures, a coupling mechanism between the curvature of the trajectory and the microscopic processes is required. We propose a stochastic model, based on the known details of polymerizing actin networks, that incorporates such a coupling. Our stochastic simulation includes nucleation, dissociation, polymerization, and capping of actin filaments. To allow for curved trajectories (in two dimensions), we allow the filament density to vary in one direction along the bead surface. Nucleation (by the Arp2/3 complex, which is activated by surface-bound N-WASP) is restricted to one-half of the bead surface and occurs with equal probability (rate constant $k_{\mathrm{n}}$ ) all over the hemispherical surface (below, we will consider a case where nucleation is inhomogeneous). Experimental evidence shows that the actin comet tail is firmly attached to the bead surface, indicating that least part of the actin filaments is linked to the surface (Cameron et al. 2001; Marcy et al. 2007). To account for this attachment, we follow (Mogilner and Oster 2003), where it was argued that an actin filament is attached to the surface when it is nucleated, associated to the proteins on the bead surface in a complex with Arp2/3 and N-WASP. After some time, the filament dissociates from this complex (with rate constant $k_{\mathrm{d}}$ ) and is able to grow and push against the bead surface, until it is capped by the capping protein gelsolin (with rate constant $k_{\mathrm{c}}$ ) and loses contact with the surface. At every instant, there is a population of attached filaments that have just been nucleated and a population of free filaments that grow. The attached filaments are in tension, because the link between the bead and the filament is stretched as the bead moves forward, resulting in a force $f_{i}^{a}=k x_{i}$ resisting bead movement. Here, $k$ is the spring constant of the attachment link and $x_{i}$ is the extension of link $i$ (equal to the trajectory length of that particular link from the moment that the link is generated). At the same time, the dissociated, growing filaments are in compression and generate the propulsion force. We assume that the filaments can be modeled as elastic rods with a bending rigidity $k_{\mathrm{f}}$. The bending rigidity depends on the length of the filament, but for simplicity we assume here that $k_{\mathrm{f}}$ is equal for all filaments. The elastic force exerted by a compressed filament is $f_{i}^{g}=k_{\mathrm{f}} \Delta_{i}$, where $\Delta_{i}$ is the distance between the equilibrium (free) position of the tip of filament $i$ and the bead surface $\left(\Delta<0\right.$ and $f^{g}<0$ for compressed filaments). If $\Delta_{i}>0$, the filament doesn't touch the surface and $f_{i}^{g}=0$. As a result of the pushing and pulling forces exerted by all attached and growing filaments, the bead moves towards an equilibrium position in which the sum of forces equals zero, as well as the sum of moments (we assume that all forces are in the forward direction): $\sum_{i} f_{i}^{a}+\sum_{j} f_{j}^{g}=0$ and $\quad \sum_{i} f_{i}^{a} r_{i}+\sum_{j} f_{j}^{g} r_{j}=0$, where $r_{i}$ is the location of the filament in the direction perpendicular to the direction of movement.

The dissociation rate $k_{\mathrm{d}}$ of filaments from the bead surface depends on the force acting on the link: pulling on a bond lowers the activation barrier and enhances dissociation. This has been observed experimentally by Marcy et al. (2004), who showed that the comet tail could be detached from the bead by pulling on it. The actual relation between the dissociation rate and the force depends on the form of the potential associated with the link, but in many cases it can be approximated by an exponential relation (Evans and Ritchie 1999)

$k_{\mathrm{d}, i}=k_{\mathrm{d}, 0} \exp \left(f_{i}^{a} / f_{l}\right)$

where $k_{\mathrm{d}, 0}$ is the dissociation rate for an unloaded link and $f_{l}$ is the strength of the attachment link. The polymerization rate constant $k_{+}$of actin filaments may also depend on the force acting on the filament (Mogilner and Oster 2003), but for simplicity we assume here that the polymerization rate is constant.

We analyze the model by performing stochastic simulations, using the algorithm of Gillespie (1977). In every simulation step, a new filament can nucleate somewhere on the bead surface, or existing filaments can detach, polymerize, or be capped, with relative probabilities given by the respective rate constants. After each microscopic reaction, the bead position and orientation are updated (by translation and rotation) to restore mechanical equilibrium, and the forces acting on the attached and free filaments are recalculated. The values for the different parameters used in the simulations are listed in Table 1. Values for $k_{+}$(Pollard 1986) and $C$ (Paluch et al. 2006) were obtained from literature data, and $\delta, k_{\mathrm{d}, 0}, k_{\mathrm{c}}, k$, and $f_{l}$ were estimated in Mogilner and Oster (2003). For the bending rigidity $k_{\mathrm{f}}$ of an actin filament, we use $0.3 \mathrm{pN} / \mathrm{nm}$, which corresponds to a filament length of a few hundred nanometers (Mogilner and Oster 1996), and for the

Table 1 Parameters used in the simulation

\begin{tabular}{lll}
\hline Symbol & Definition & Value \\
\hline$k_{\mathrm{n}}$ & Nucleation rate per unit area & $0.8 \mathrm{~s}^{-1} \mu \mathrm{m}^{-2}$ \\
$k_{\mathrm{d}, 0}$ & Free detachment rate & $0.1 \mathrm{~s}^{-1}$ \\
$k_{+}$ & Polymerization rate & $10 \mathrm{~s}^{-1} \mu \mathrm{M}^{-1}$ \\
$C$ & Actin monomer concentration & $1 \mu \mathrm{M}$ \\
$k_{\mathrm{c}}$ & Capping rate & $0.15 \mathrm{~s}^{-1}$ \\
$\delta$ & Effective monomer size & $2.7 \mathrm{~nm}$ \\
$k$ & Spring constant of attachment link & $3 \mathrm{pN} \mathrm{nm}^{-1}$ \\
$k_{\mathrm{f}}$ & Spring constant of actin filament & $0.3 \mathrm{pN} \mathrm{nm}$ \\
$f_{l}$ & Effective strength of attachment bond & $6 \mathrm{pN}$ \\
\hline
\end{tabular}


nucleation rate $k_{\mathrm{n}}$ we use $0.8 \mathrm{~s}^{-1} \mu \mathrm{m}^{-2}$. With these parameters, the filament density is around $100 \mu \mathrm{m}^{-2}$, about 5-10 percent of which is attached. The resulting average bead velocity is $1.5 \mu \mathrm{m} \mathrm{min}{ }^{-1}$, similar to the experimental velocities.

Figure 1e displays simulated particle trajectories for this parameter set for three different bead sizes. The curvature of the simulated trajectories is analyzed in the same way as the experimental trajectories. The resulting curvature distribution is shown in Fig. $2 \mathrm{c}$ and $\mathrm{d}$, together with the experimental data. Although the absolute values of the curvatures differ between experiments and simulations (Fig. 2b, see also below), the shape of the simulated curvature distribution is in excellent agreement with the experimental curve. The non-Gaussian shape of the distribution is produced in the simulations as a result of the coupling between the force acting on the attachment bonds and the detachment rate (1). Indeed, if this coupling is turned off [by taking $f_{l}$ infinitely large in (1)], the simulated curvature distributions become Gaussian, as would be expected for independent, additive random processes. The physical reason for the non-Gaussian curvature distribution and the relatively high frequency of large curvatures is a positive feedback mechanism. When the bead bends off to the right, attachment bonds on the left (in the outer bend) are more strongly stretched than those on the right (in the inner bend). According to (1), this leads to faster detachment on the left and thus to more detached, pushing filaments on the left and an increase of the torque that induces curvature to the right. This positive feedback explains the relatively high propensity for high curvatures observed in the experiments and simulations.

Figure $2 \mathrm{~b}$ displays the root-mean-square curvature as a function of bead size (red diamonds). Like in the experiments, the mean curvature decreases with increasing bead radius, probably because larger beads have more filaments, so that fluctuations are relatively less important. However, the effect of the radius is much stronger in the simulations than in the experiments: between $R=1 \mu \mathrm{m}$ and $R=3 \mu \mathrm{m}\langle\kappa\rangle$ decreases by a factor of 40 in the simulations and by a factor of 1.5 in the experiments. A possible explanation for this difference may be that the reaction rates on the bead surface are inhomogeneous, due to slow diffusion of proteins through the comet tail. Such diffusion limitation is indeed suggested by our observation that the speed of the beads decreases by about a factor of two when $R$ increases from 1.5 to $3 \mu \mathrm{m}$ (see Paluch et al. 2006). Due to hindered diffusion through the dense actin network, a concentration gradient of proteins arises in the comet tail, with the lowest concentration in the middle of the tail. This effect is much stronger for large beads than for small ones, because of the larger diffusion distance. The Arp $2 / 3$ concentration gradient leads to a nucleation probability that is lower in the middle of the bead than at the edges, which leads, in turn, to a lower filament density in the middle. Indeed, about $80 \%$ of the comet tails for the $3 \mu \mathrm{m}$ beads appeared hollow (see Fig. 1c). To test the effect of inhomogeneous nucleation on the shape of the trajectories, we did simulations with an inhomogeneous nucleation probability, keeping all other reaction rates homogeneous. At steady state, the diffusive flux of free Arp2/3 complex $J_{\mathrm{D}}=D \partial C_{n} / \partial r$ equals the monomer consumption per unit area $J_{\mathrm{R}}=k_{\mathrm{n}}^{\prime} C_{n}$ (with $C_{n}$ the concentration of free $\operatorname{Arp} 2 / 3$ complex), so that the concentration of Arp2/3 decreases exponentially from the edges towards the middle of the tail: $k_{\mathrm{n}}(r) \sim C_{n} \simeq C_{n}^{\infty} \exp \left[(r-R) / \xi_{\mathrm{D}}\right]$, with the diffusive layer $\xi_{\mathrm{D}}=D / k_{\mathrm{n}}^{\prime}$. In Fig. $2 \mathrm{~b}$, we plotted simulation results for $\xi_{\mathrm{D}}=0.5 \mu \mathrm{m}$ (green triangles). Clearly, inhomogeneous nucleation leads to a radius-dependence that is closer to the experimental trend, although still not completely the same. In practice, of course, also the polymerization and capping rates will be affected by protein diffusion, each with their own diffusive layer. However, a detailed study of these effects is beyond the scope of this study.

Experimentally, we observe that the bead velocity gradually decreases in time (to about $20 \%$ of the initial velocity after $8 \mathrm{~h}$ ). Interestingly, the mean curvature is hardly affected (Fig. 2a). The decrease of the velocity is probably due to aging of the beads and the proteins in the medium, leading to a decrease of the different reaction rates. Indeed, if we decrease all rate parameters in our model by the same factor, the simulations reproduce the experimental findings: the average velocity decreases, while the curvature distribution is unmodified.

In conclusion, the actin-based movement of biomimetic colloids is characterized by a non-Gaussian curvature distribution. The relatively high probability of large curvatures may be advantageous for bacteria and vesicles, for example, for avoiding obstacles. A stochastic kinetic model nicely reproduces the non-Gaussian shape of the experimental curvature distribution. This work thus provides strong support for a force-dependence of the microscopic reactions involved in actin-based motility. In the present paper, only the detachment rate was assumed to depend on the force, but in practice also the polymerization rate could be force-dependent, leading to an additional positive feedback mechanism.

Acknowledgments We acknowledge financial support of the Alexander von Humboldt Foundation (Germany) and European Community STREP Active Biomics. J. vdG. acknowledges Human Frontier Science Program Organization for financial support. We thank Dominique Didry, Diep Le and Marie-France Carlier for providing purified motility proteins. 
Open Access This article is distributed under the terms of the Creative Commons Attribution Noncommercial License which permits any noncommercial use, distribution, and reproduction in any medium, provided the original author(s) and source are credited.

\section{References}

Boukellal H, Campas O, Joanny J-F, Prost J, Sykes C (2006) Soft Listeria: actin-based propulsion of liquid drops. Phys Rev E 69:061906

Cameron LA, Svitkina TM, Vignjevic D, Theriot JA, Borisy GG (2001) Dendritic organization of actin comet tails. Curr Biol 11:130-135

Cameron LA, Robbins JR, Footer MJ, Theriot JA (2004) Biophysical parameters influence actin-based movement, trajectory, and initiation in a cell-free system. Mol Biol Cell 15:2312-2323

Cameronv LA, Footer MJ, Van Oudenaarden A, Theriot JA (1999) Motility of acta protein-coated microspheres driven by actin polymerization. Proc Natl Acad Sci USA 96:4908-4913

Carter BC, Shubeita GT, Gross SP (2005) Tracking single particles: a user-friendly quantitative evaluation. Phys Biol 2:60-72

Evans E, Ritchie K (1999) Strength of a weak bond connecting flexible polymer chains. Biophys J 76:2439-2447

Giardini PA, Fletcher DA, Theriot JA (2003) Compression forces generated by actin comet tails on lipid vesicles. Proc Natl Acad Sci USA 100:6493-6498

Gillespie DT (1977) Exact stochastic simulation of coupled chemicalreactions. J Phys Chem 81:2340-2361

Kreyszig E (1991) Differential geometry. Dover, New York

Loisel TP, Boujemaa R, Pantaloni D, Carlier M-F (1999) Reconstitution of actin-based motility of Listeria and Shigella using pure proteins. Nature 401:613-616

Marcy Y, Prost J, Carlier M-F, Sykes C (2004) Forces generated during actin-based propulsion: a direct measurement by micromanipulation. Proc Natl Acad Sci USA 101:5992-5997
Marcy Y, Joanny J-F, Prost J, Sykes C (2007) Probing friction in actin-based motility. New J Phys 9:431

Mogilner A, Oster G (1996) Cell motility driven by actin polymerization. Biophys J 71:3030-3045

Mogilner A, Oster G (2003) Force generation by actin polymerization II: the elastic ratchet and tethered filaments. Biophys J 84:15911605

Paluch E, Van der Gucht J, Joanny J-F, Sykes C (2006) Deformations in actin comets from rocketing beads. Biophys J 91:3113-3122

Pantaloni D, Le Clainche C, Carlier M-F (2001) Cell biologymechanism of actin-based motility. Science 292:1502-1506

Pollard TD (1986) Rate constants for the reactions of ATP-actin and ADP-actin with the ends of actin-filaments. J Cell Biol 103:2747-2754

Rutenberg AD, Grant M (2001) Curved tails in polymerization-based bacterial motility. Phys Rev E 6402:021904

Shaevitz JW, Fletcher DA (2007) load fluctuations drive actin network growth. Proc Natl Acad Sci USA 104:15688-15692

Shenoy VB, Tambe DT, Prasad A, Theriot JA (2007) A kinematic description of the trajectories of Listeria monocytogenes propelled by actin comet tails. Proc Natl Acad Sci USA 104:82298234

Theriot JA, Mitchison TJ, Tilney, LG Portnoy DA (1992) The rate of actin-based motility of intracellular Listeria monocytogenes equals the rate of actin polymerization. Nature 357:257-260

Upadhyaya A, Chabot JR, Andreeva A, Samadani A, Van Oudenaarden A (2003) Probing polymerization forces by using actinpropelled lipid vesicles. Proc Natl Acad Sci USA 100:45214526

Van der Gucht J, Paluch E, Plastino J, Sykes C (2005) Stress release drives symmetry breaking for actin-based movement. Proc Natl Acad Sci USA 102:7847-7852

Wiesner S, Helfer E, Didry D, Ducouret G, Lafuma F, Carlier M-F, Pantaloni D (2003) A biomimetic motility assay provides insight into the mechanism of actin-based motility. J Cell Biol 160:387398 